\title{
Phase II Final Report Computer Optimization of Electron Guns Contract DE-FG02-06ER86276
}

For period June 28, 2006 through August 8, 2010

\author{
R. Lawrence Ives, Principal Investigator \\ Calabazas Creek Research, Inc. \\ 690 Port Drive, San Mateo, CA 94404 \\ (650) 312-9575, Fax: (650) 312-9527 \\ RLI@CalCreek.com \\ www.calcreek.com
}

\section{SBIR/STTR RIGHTS NOTICE}

These SBIR/STTR data are furnished with SBIR/STTR rights under Grant No. DE-FG0206ER86267. For a period of four (4) years after acceptance of all items to be delivered under this grant, the Government agrees to use these data for Government purposes only, and they shall not be disclosed outside the Government (including disclosure for procurement purposes) during such period without permission of the grantee, except that, subject to the foregoing use and disclosure prohibitions, such data may be disclosed for use by support contractors. After the aforesaid four year period, the Government has a royalty free license to use, and to authorize others to use on its behalf, these data for Government purposes, but is relieved of all disclosure prohibitions and assumes no liability for unauthorized use of these data by third parties. This Notice shall be affixed to any reproductions of these data in whole or in part. 


\section{Introduction}

This program was tasked with extending computer optimization to the design of complex, 3D electron gun design. The specific tasks are:

- Identify optimization processes, constraints, and goal functions for generalized, 3D electron beam devices,

- Translate all computer algorithms, including the control program, design table modification, optimization routines, and manipulation of output data into $\mathrm{C}++$, and integrate into Beam Optics Analysis

- Develop an advanced graphical user interface for setting up and performing optimized design

- Enhance Beam Optics Analysis to increase efficiency and reduce computation time

- Test proper operation using a variety of challenging electron device designs.

Progress was completed on all these tasks, as described below. The first task was primarily addressed at North Carolina State University (NCSU), the non-profit research institute associated with this program. Translation of the algorithms was a joint effort between NCSU and Calabazas Creek Research, Inc. (CCR). CCR was responsible for the graphical user interface (GUI) development and the enhancement of Beam Optics Analysis (BOA). Testing was performed at NCSU and CCR.

During the first year, efforts initially focused on optimization routines for a generalized doubly convergent electron gun. This involved elimination of spiraling as the beam is compressed toward the axis of the magnetic field. This successful effort demonstrated the power of optimization in achieving a design that had not previously been accomplished. Results of this effort were presented at the 2008 [1], 2009 [2], and 2010[3] International Vacuum Electronics Conferences. A patent application was also submitted on the double convergent gun design and is currently under review by the U.S. Patent Office [5].

A major advanced occurred when a magnetics solver was integrated into BOA during the final period of the program. This capability allows optimized design of the electrostatics and magnetostatics with the beam optics design. The program developed optimization algorithms using this new capability.

The GUI integrates optimized design of the Pierce gun capability developed in Phase I with the advances made in the Phase II program. These optimization algorithms are completed and tested. A considerable amount of work was involved in data handling and transfer between the electrostatic/beam analysis and the magnetics analysis, as each simulation used the same geometry file but generated different meshes and required different input. It was necessary to provide interpolation of results from one mesh to another. The final design allows seamless transfer of results between the two analyses within the same case file.

BOA was also enhanced to include parallelization to dramatically reduce computation time. This is especially critical for complex designs involving multiple or distributed electron beams. The parallelization effort includes calculation of beam trajectories and meshing. This research was presented at the 2008 and 2009 IVECs [6]. 
The remaining sections describe the results for each task.

\section{Optimization processes, constraints, and goal functions for generalized, 3D electron beam devices}

An integral component during the first year of Phase II program was to develop goal functions for achieving specified magnetic field profiles in axially symmetric magnetic circuits using solenoids and iron. In addition, the Phase I effort was extended to include optimization of specific trajectories from sheet beam guns. This required adding capability to identify and track specific electron trajectories and direct them to specified locations in the problem domain.

1. Magnetic Circuit Design. In this design, the goal was to determine a solenoid current value that produces a magnetic field value 2.5 times the Brillouin value at the center of the magnet, located at $\mathrm{z}=110.0 \mathrm{~cm}$. That is, the goal was to obtain a yield strength of $0.035 \mathrm{~T}$ using the following goal function

$$
J=\left(B_{z=110}-0.035 T\right)^{2}
$$

In another design configuration, the suite of software design tools sought to obtain a desired field strength at the cathode $\mathrm{z}$ location, $\mathrm{z}=-60.0 \mathrm{~cm}$ by varying the poll piece diameter on the magnet. The desired field strength at the cathode was 70.23 Gauss, which was obtained from the relation (Busch's Theorem)

$$
B_{k} A_{k}=B_{c} A_{c}
$$

The goal function that described this desired objective was given by

$$
J=\left(B_{z=-60}-70.23 G\right)^{2} .
$$

More recent work focused on achieving specified magnetic field profiles by manipulating magnetic circuit geometry and coil currents. The magnetic field in a klystron or TWT, for example, often increases at the output end of the device to more tightly confine electrons whose energy has been reduced to generate the output RF power. The intent was to take a desired magnetic field profile requested by the user and attempt to achieve that profile by manipulating the coil geometry, polepiece geometry and coil currents.

Figure 1shows a magnetic circuit used to test the procedure. This design includes input and output polepieces, six magnet coils, and cutouts for the output waveguide. Simulation/ optimization results are shown on the right, where the green curve represents the desired field and the blue curve indicates the field obtained by the process. As can be seen, the actual field achieves the best fit available with the geometrical constraints. This design would easily satisfy the performance requirement.

The goal functions were implemented into BOA, and the user interface upgraded accordingly. This capability is now available for users. 

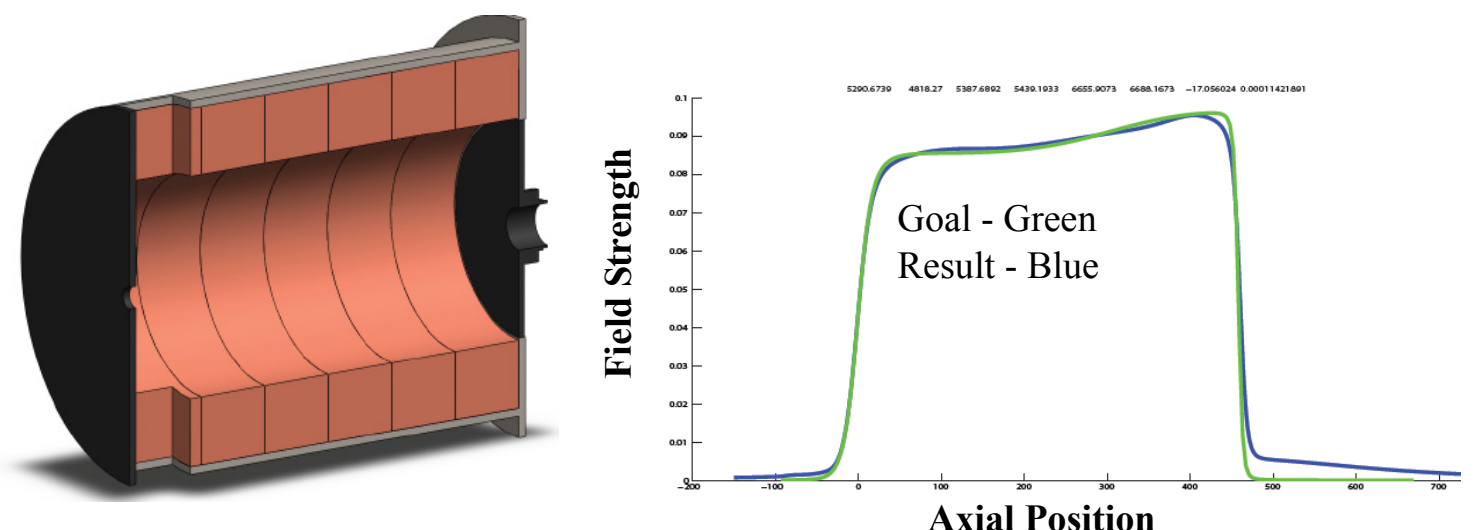

Figure 1. Six coil magnet for an RF source (left) and optimized magnetic field (right)

2. Sheet Beam Gun Design. Here, the initial focus was to design a sheet beam cathode to achieve a specified current, along with specification of the $\mathrm{z}$ location of the beam minimum and the thickness of the beam at this location. The appropriate goal function was s given by

$$
J=10(I-11 A)^{2}+\left(y_{j}-0.7\right)^{2}+\left(C A S+5-z_{j}\right)^{2}
$$

where $y_{j}$ is the thickness of the beam at $j$, the beam minimum, CAS is the cathode-anode spacing, and $\mathrm{z}_{\mathrm{j}}$ is the location of the beam minimum in the $\mathrm{z}$ plane. The goals are $11.0 \mathrm{~A}$ for the cathode current, $0.7 \mathrm{~mm}$ thickness at the beam minimum, and to achieve the minimum thickness $5.0 \mathrm{~cm}$ into the beam tunnel.

This particular task was modified during the second year of the program. Results from Stanford Linear Accelerator Center (SLAC) indicated that sheet beam klystron were subject to serious beam tunnel instabilities between cavities. As a result, interest in sheet beam devices dramatically dropped, at least for applications of importance to the Department of Energy.

This task shifted to focus more on multiple beam guns. Most new development for high power RF sources are centered around multiple beam devices, both klystrons and inductive output tubes (IOTs). The initial effort was to reduce the beam ripple about the local beam axis for each beam. Hence, an objective was to minimize radial and angular velocities as defined by the following goal function

$$
J=\sum \alpha \frac{d \theta}{d z}+(1-\alpha) \frac{d r}{d z},
$$

where the sum is across particles and slices in the z-plane and $\alpha$ is a weighting parameter, possibly dependent on the $\mathrm{z}$ location of the slice taken. A key issue is the radial variation about the local axis of the individual beams, which is different from the axis of the device. 
This goal function played a crucial role in the development of the double convergent multiple beam gun, which will be described in more detail in Section 6 .

\section{Translate all computer algorithms, including the control program, design table modification, optimization routines, and manipulation of output data into $\mathrm{C}++$, and integrate into Beam Optics Analysis}

NCSU employs MATLAB to initially develop and test computer algorithms for optimization. This allows rapid modification and testing of different approaches and techniques. 'Canned' routines are available for various optimization techniques, including Nelder-Mead and Implicit Filtering, the primary approaches used in this program.

To complete the development, these routines were integrated into the BOA deliverable executable. BOA is primarily constructed using $\mathrm{C}++$ for flexibility and extensibility. The commercial version of BOA is distributed as a downloadable executable from the CCR web site at www.calcreek.com. Therefore, the Nelder-Meade and Implicit Filtering optimization routines were integrated into this executable. After the optimization routines were integrated, the control program to perform the optimization process was integrated. This use the input parameter specified in the optimizer GUI, described in the next section.

\section{Develop an advanced graphical user interface for setting up and performing optimized design}

The GUI for optimization was completed, though additional goal functions and capability are continually being added. Users can select either single case simulation or optimizer. Figure 2 shows the window displayed for single case execution. The optimizer menu is

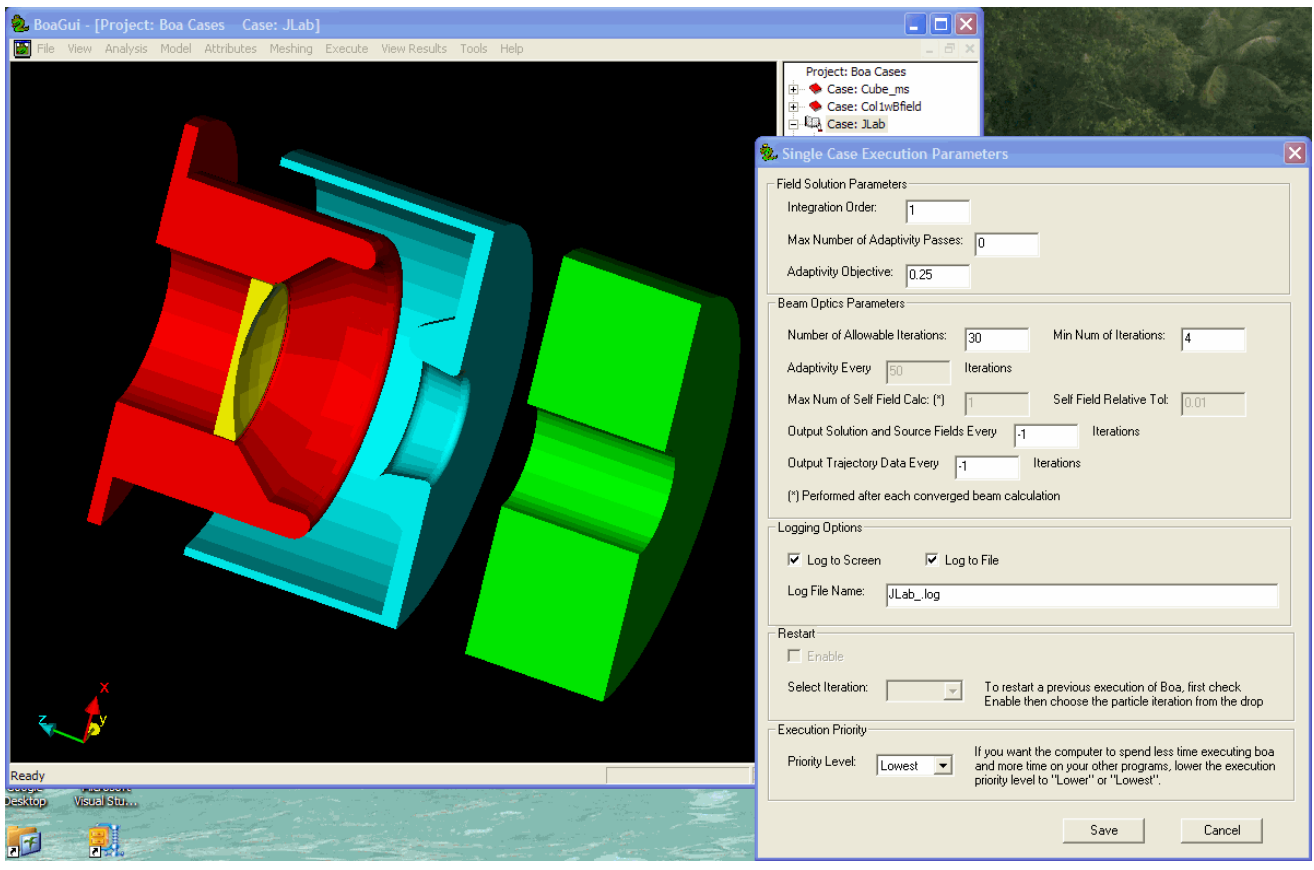

Figure 2. Single case menu to specify execution parameters. 
only enabled after the user completes the input requirements for the single case simulation. In the single case window, the user can select interpolation order, with or without adaptivity, maximum number of allowable particle iterations, convergence criteria, etc.

After the user completes the specification of single case execution parameters, the optimizer menu, shown in Figure 3, is enabled. Note that this optimization only applies to electrostatic and beam analysis. There is an independent optimization GUI for magnetics analysis. In this type of analysis, users can select four types of optimizations: beam current, diameter, ripple and electric field gradient. Appropriate parameters for each optimization type are located to the right and enabled only for the selected type. Users can choose either Nelder-Mead or Implicit optimization. Further parameters for geometry optimization can also be specified. Finally, execution parameters, including restart options, are available.

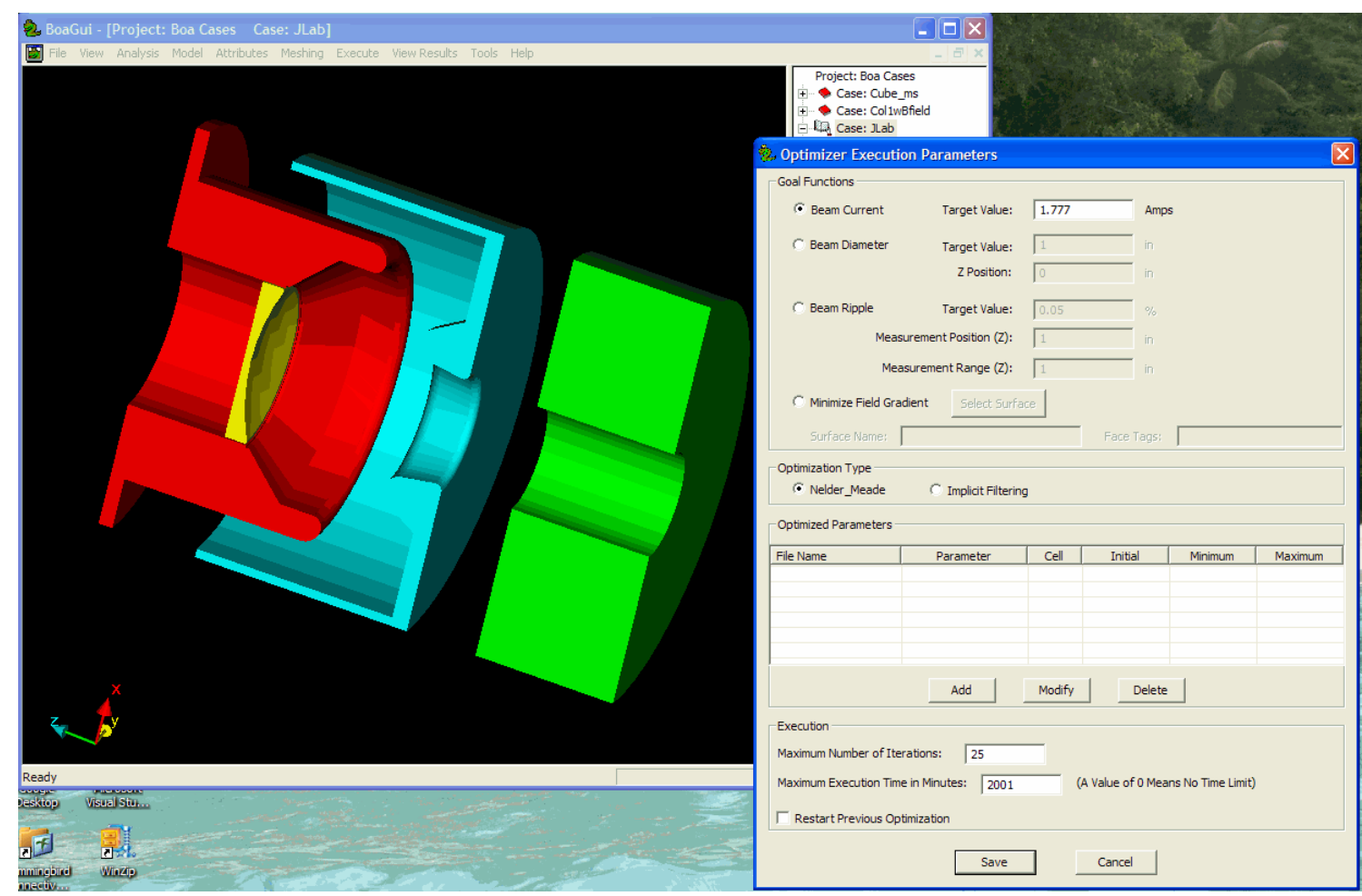

Figure 3. Optimizer menu to specify optimization parameters.

\section{Enhance Beam Optics Analysis to increase efficiency and reduce computation time}

As a result of this program, BOA includes a fully functional, nonlinear, magnetostatic solver and can simulate most all coil geometries. The nonlinear solver uses Newton's method with analytic Jacobian to achieve super linear convergence, as shown by Figure 4. Figure 4 is a semilog plot of residual history of a coax with the nonlinear material sandwiched between the conductors. 
The cotree technique was initially used to solve the singular system produced by the vector finite element formulation when coils are present. This method provided good results but was computationally slow. A faster method to handle singularities was required. The graphical user interface was developed to allow input current for coils and add nonlinear materials, either by table selection or using mathematical expressions.

Without imposing a gauge condition, the vector finite element method used

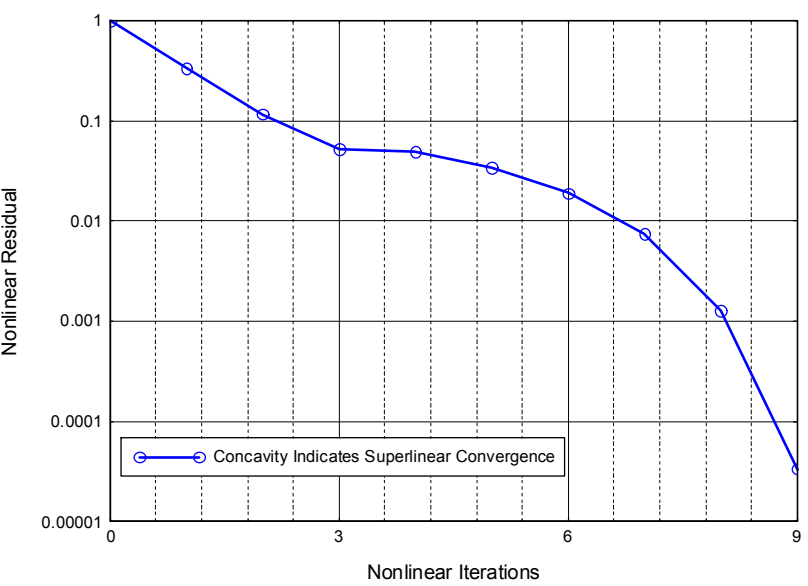

Figure 4. Newton iteration for coaxial problem to discretize the Curl-Curl equations in magnetostatics produced a singular system. The cotree technique was initially implemented to numerically enforce the Coulomb gauge. Extensive literature research indicated that imposing the Coulomb gauge was unnecessary, and a conjugate gradient, iterative, linear, equation solver would solve such a singular system. Further investigation of the BOA code revealed a deficiency in the linear solver. This in-house implementation of the conjugate gradient solver did not include a proper and robust preconditioner to correctly handle singularities. By switching to the linear equation solver provided by PETSc (the Portable, Extensible Toolkit for Scientific computation,), which was developed and maintained by Argonne National Laboratory, rendered the cotree scheme unnecessary and accelerated the solver considerably. The magnetostatic field solver is now fully optimized to handle linear and nonlinear materials, including the user interfaces for coils and permanent magnets (see Figure 5). It is also fully parallelized with OpenMP.

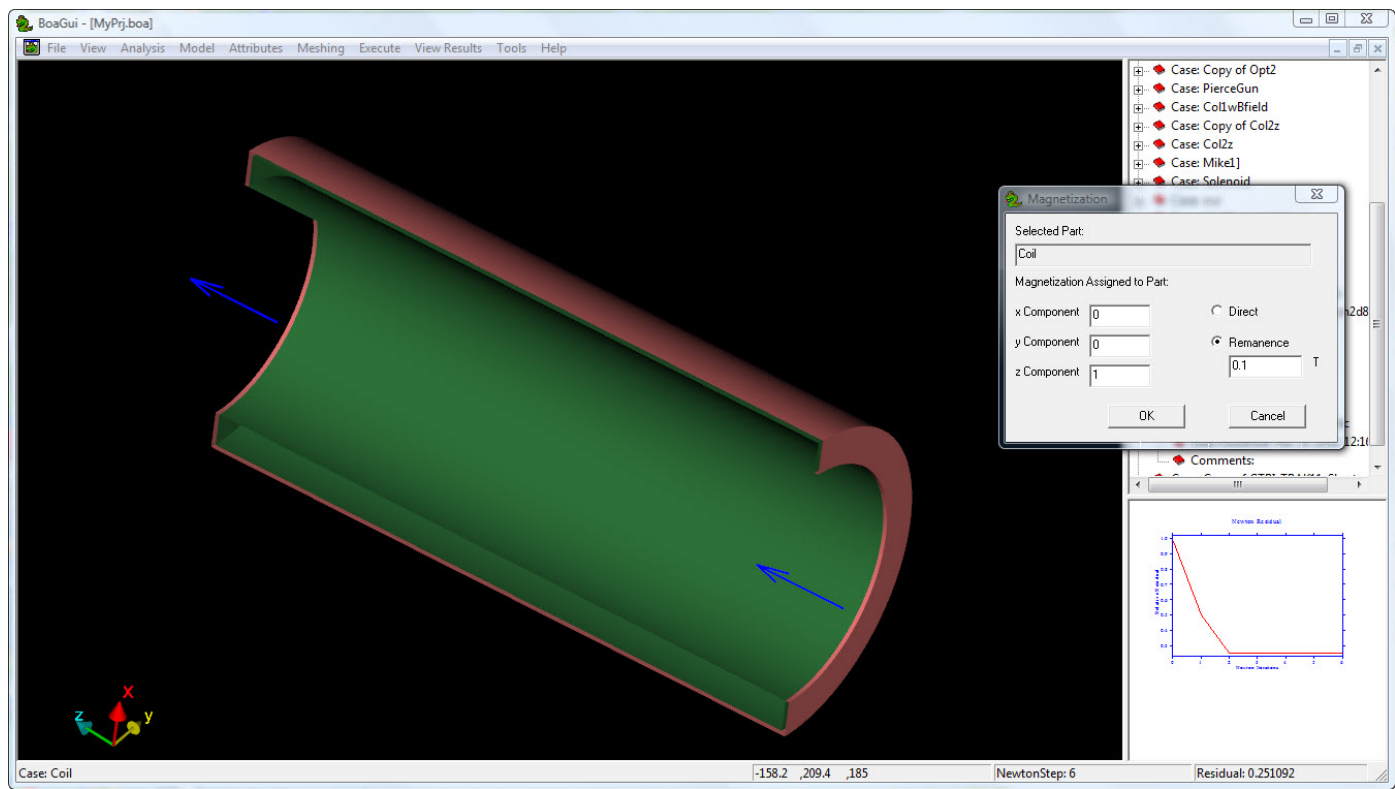

Figure 5. Typical graphical user interface for magnetics analysis 
Adaptivity for both electrostatic and magnetostatic is also parallelized fully taking advantage of multi-core systems.

Parallelization of BOA is proceeding extremely well. BOA uses Simmetrix Partitioned Mesh to parallelize the mesh generator. For a mesh of 114,646 tetrahedra, 229,753 faces, 134,296 edges and 19,190 vertices, the serial version takes 43 seconds to generate in comparison to 15.3 seconds for the parallel version on a $2 \mathrm{GHz}$ Intel Core Duo laptop. OpenMP allows parallelization of the electrostatic solver, field smoothing, adaptivity and particle pusher. An electrostatic beam simulation without adaptivity and a 114,646 tetrahedra mesh converges after 21 particle iterations and 1,732 seconds. to complete with the serial version. The parallel version completes this computation in only 1026 seconds.

\section{Test proper operation using a variety of challenging electron device designs}

Designs were tested as the optimization routines were generated. Routines for Pierce electron guns were tested and are operational, as are magnetic circuits. During this task significant technological breakthroughs were achieved with advanced multiple gun designs, both for klystrons and IOTs. The optimization capability allowed design of structures that were previously impractical.

The most dramatic demonstration was development of a doubly convergent multiple beam electron gun. This device had previously been assumed to be impossible due to the spiraling that occurs when electron beams are compressed in a magnetic field. All multiple beam guns designed to date use non-convergent or singly convergent electron beams. This includes the many designs developed in the former Soviet Union and the higher power devices currently available from Thales Electron Devices, Toshiba, and Communications \& Power Industries, Inc. CCR built a single convergent multiple beam klystron at X-Band and designed several singly convergent beams for other devices [8]. CCR also has two patents for singly convergent multiple beam guns $[9,10]$.

The doubly convergent device was particularly challenging because of the requirement to satisfy canonical angular momentum as the individual electron beams are compressed about their local axis as well as the axis of the magnetic field. As assumed, the azimuthal velocity of the beams changes as the magnetic field is changed. Consequently, beams emitted parallel to the device axis at the electron gun will spiral around the axis as they are compressed toward the axis of the device. Such beams would be impractical for an RF device.

The program used two goal functions to achieve a practical design. First, a goal function was developed that optimized the path of the electron beam through a region of the geometry. This was a 'linearity' goal function, given by

$$
\begin{aligned}
\text { Linearity } & =\sum_{i=2}^{N-1}\left(\left(\frac{y_{N}-y_{1}}{z_{N}-z_{1}} z_{i}+y_{N}-\frac{y_{N}-y_{1}}{z_{N}-z_{1}} z_{N}\right)-y_{i}\right)^{2} \\
& +\sum_{i=2}^{N-1}\left(\left(\frac{x_{N}-x_{1}}{z_{N}-z_{1}} z_{i}+x_{N}-\frac{x_{N}-x_{1}}{z_{N}-z_{1}} z_{N}\right)-x_{i}\right)^{2}
\end{aligned}
$$


This function summed the distances of the beam centers at various axial positions from the desired path. The optimizer then attempted to minimize this value. The parameters varied were the angular orientations (in radial plane and azimuthal plane) of the cathode axis with respect to the device axis. The second goal function, applied after the linearity optimization was complete, modified the cathode radius of curvature to minimize beam scallop. This goal function is given by,

$$
\text { Scall }=100 \frac{d_{\text {max }}-d_{\text {min }}}{d_{\text {max }}+d_{\text {min }}}
$$

The geometry of a single gun is shown in Figure 6. The optimization process only needed to simulate one off-axis gun of the multiple beam device. In this design, the cathode, focus electrode, and anode were maintained fixed with respect to each other. The orientation of this assembly was then modified as directed by the optimization control routine. The goal functions were evaluated after the beam achieved full magnetic compression in the simulated circuit region.

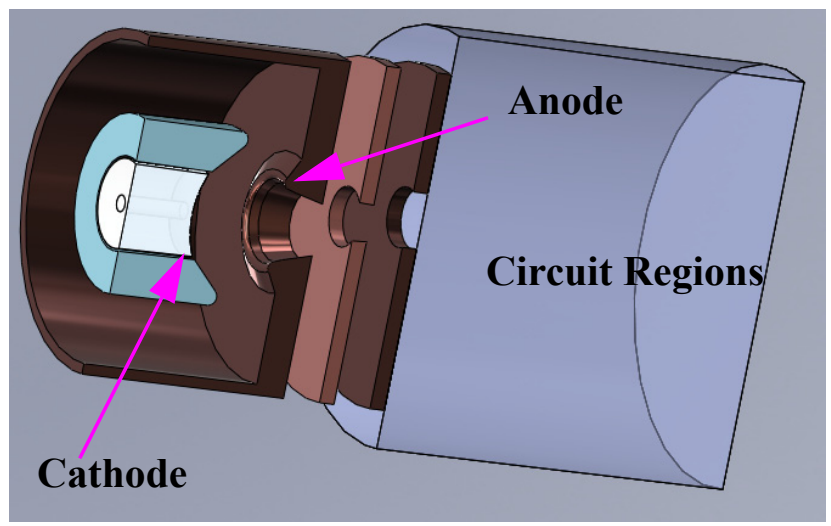

Figure 6. Solid model of one gun in doubly convergent multiple beam gun

Figure 7 shows the final result for the optimization. The cathode is located $60 \mathrm{~mm}$ from the device axis, and the beam experiences an area compression of 16:1 about it's local axis and of the beam center about the device axis. To achieve linear (non-spiraling) propagation through the circuit region, the electron gun is pointed toward the device axis and a few degrees in the azimuthal direction.

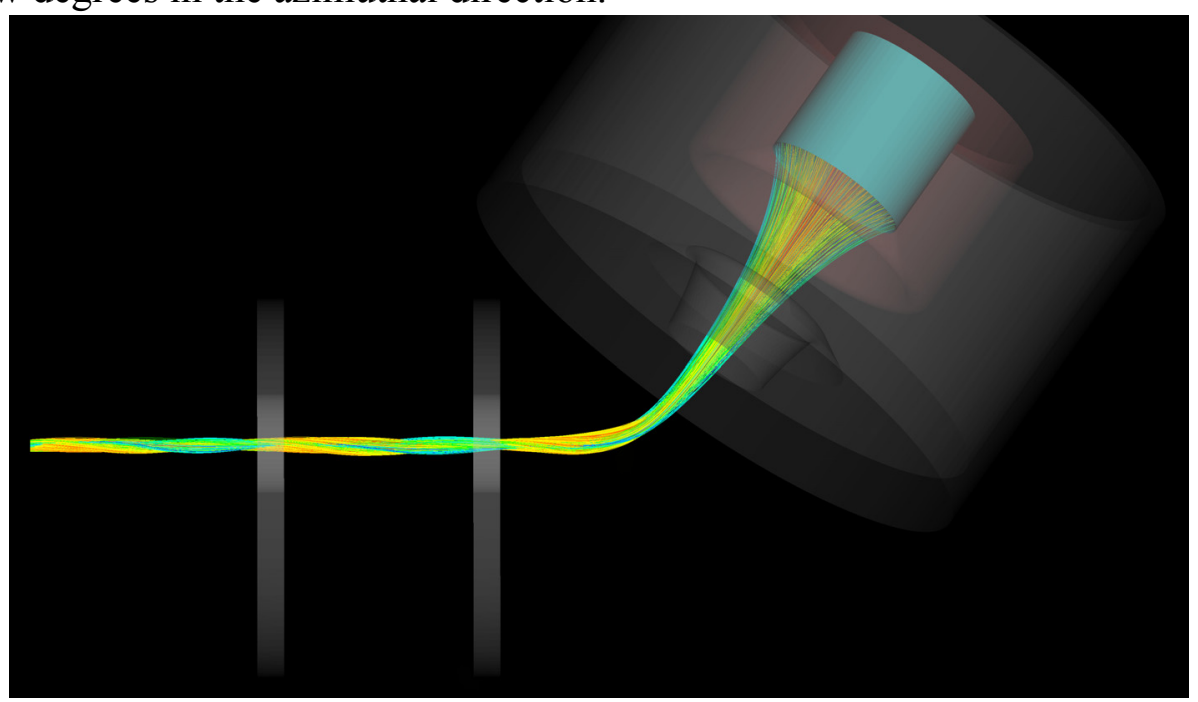

Figure 7. Simulation of optimized electron beam for a doubly convergent multiple beam gun. The center of the cathode is $60 \mathrm{~mm}$ from the device axis and the final beam is approximately $15 \mathrm{~mm}$ from the axis. 
In a final device, several of these guns would be arrayed azimuthally about the axis of the device. Figure 8 shows images of a four beam gun using this design. A complete description of the optimization routines, goal functions, design process, and results were recently published in IEEE Transactions on Plasma Science [11].

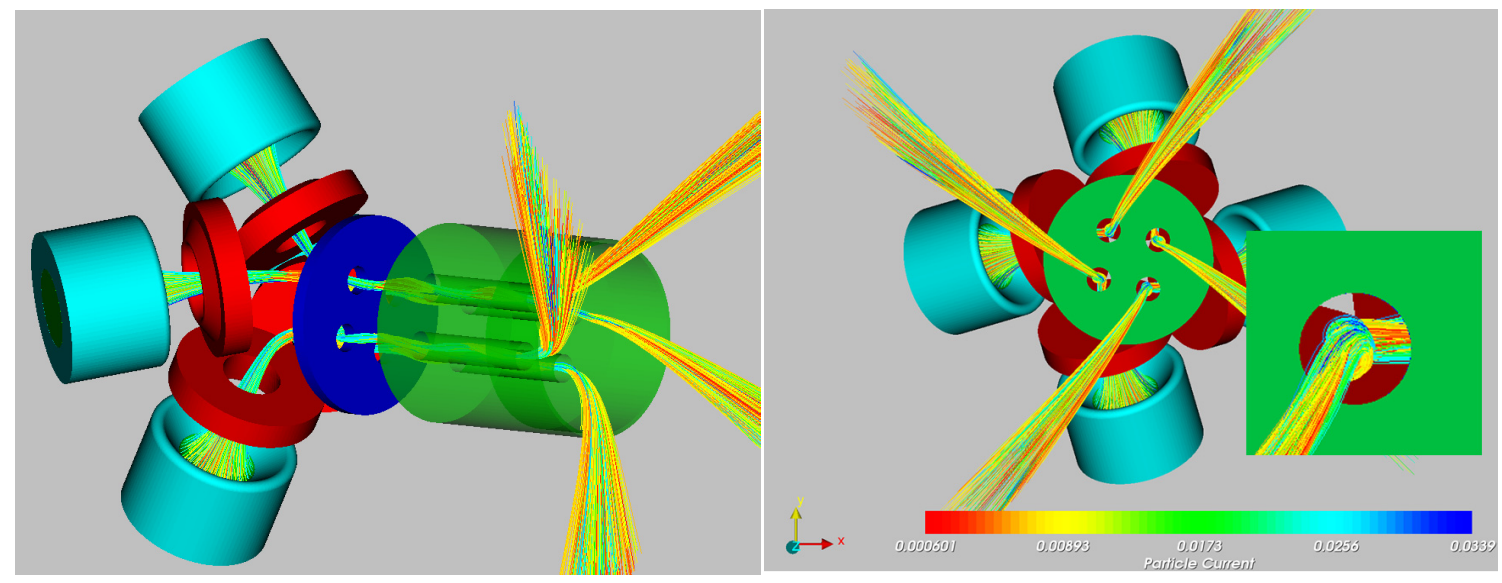

Figure 8. Views of a 4-beam, doubly convergent, multiple beam electron gun

A second application including magnetic field optimization involved development of a multiple beam IOT. The requirement in this case was to design a flat magnetic field at the off-axis location of the electron guns. This flat field was required in the presence of an input polepiece opening for electrical connections and an output polepiece providing radial dispersal of the electron beam into the collector. Figure 9 shows the beam simulation for the final design. The challenge was to achieved the desired magnetic field profile by modifying coil dimensions, coil currents and the geometry of the output polepiece.

\section{Implementation of Genetic Algorithms}

Though it was not included in the proposal, the university partner, N.C. State University, applied genetic algorithms to electron beam device design optimization. Although optimization is a very useful method, no one optimization process works well for every problem. Global search methods provide more reliability but need more function evaluations, which can be very timely. Using a local search is much quicker but less reliable due to the constraints on search space. In and effort to achieve the best of both methods, a hybrid algorithm was created by fusing a local method (Nelder-Mead) and a global method.

A genetic algorithm utilizes evolutionary strategies to optimize a population over the entire parameter space. As such, it does not require an initial guess or initial bounds like local search mechanisms, such as Nelder-Mead. The genetic algorithm written for the research utilizes a recombination mechanism, as well as two mutations, in order to gradually move the population toward the global minimum. Recombination creates a weighted average of the parameters of two individuals within the population, creating a new individual whose parameters are closer to the individual with a better cost value. However, recombination alone presents the risk of the population falling into a convex hull. Mutations add variability into the population that lead to a "crawling" effect toward 
the global minimum. One mutation adds a completely random point into the population, while the other perturbs a randomly chosen point in a random direction and radius in an attempt to move it closer to the global minimum. These mutations will invariably create an improved point in the populations, which will draw other points to it through recombination.

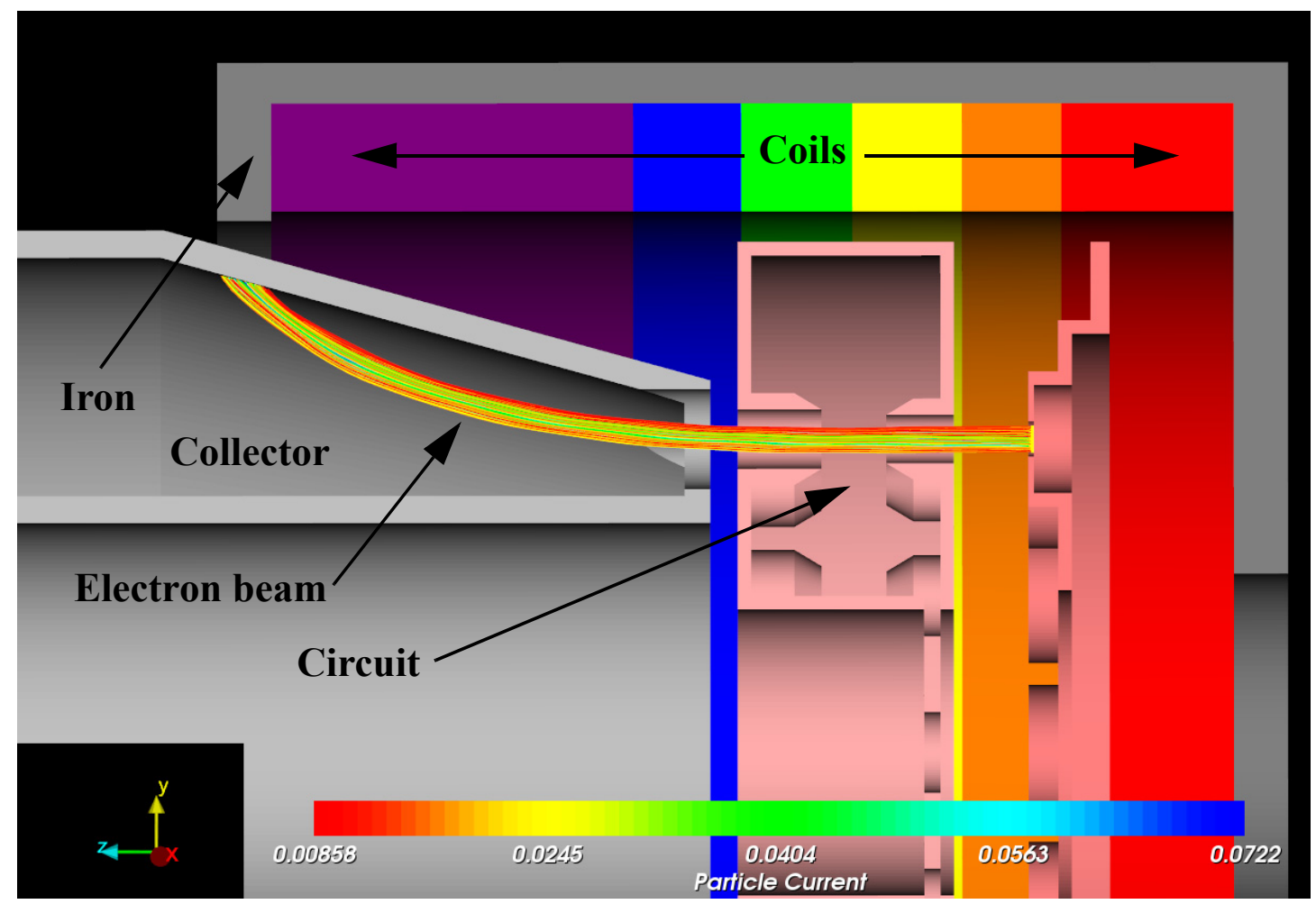

Figure 9. Electron beam propagation in optimized magnetic field for a multiple beam inductive output tube

The implementation of the genetic algorithm occurred in the context of a hybrid search algorithm. the genetic algorithm returns its population, which then gets fed to a cluster detection program. By doing so, all local minima detected by the genetic algorithm can be tested for the possibility of containing the global minimum. After clusters have been detected, the local search method, Nelder-Mead, is run with one instance in each of the detected clusters. The Nelder-Mead algorithm is run with a threshold value that is $1 \%$ of the threshold value of he genetic algorithm. Once all of the instances of Nelder-Mead have converged, the optimal points in each instance are compared, and the point with the lowest cost function evaluation is determined to be the global minimum of the function.

The optimization of the IOT magnetic field described in the last section was designed using the both the previous method (Nelder-Mead) and the hybrid method including the genetic algorithm. The hybrid method achieved a better result with fewer evaluations than either the Nelder-Mead or genetic algorithms by themselves.

It is anticipated that the hybrid method will be integrated into BOA in the next year. 


\section{Summary}

All tasks proposed were successfully completed. In addition research on genetic algorithms identified an hybrid approach that should lead to improved performance.

Results of the Phase II program were presented at the International Vacuum Electronics Conference in Monterey, CA in April 2008 [1, 6], in Rome in April 2009 [4] and again in Monterey in 2010 [3]. Papers were also presented at the Infrared and Millimeter-wave conferences in 2009 and 2010 [12, 13] and at the International Conference on Plasma Science in 2010 [14]. In addition, results from the program were published in IEEE Transactions on Plasma Science in February 2008 [7], May 2009 [15] and June 2010 [11]. An patent application for the doubly convergent electron gun is currently under review by the U.S. Patent Office [5].

Beam Optics Analyzer is currently being marketed internationally by CCR. The capabilities integrated during this program significantly improved the capability and market for this code. It is also providing CCR with a design capability that exceeds that of any other small business in this field, as well as most other large corporations.

\section{References}

[1] R. Lawrence Ives, Thuc Bui, Michael Read, John David, Adam Attarian, Hien Tran, "Computer Optimized Design of Electron Guns," 2008 IEEE International Vacuum Electronics Conference, Monterey, CA, April 2008, pp 453-454.

[2] R. L. Ives, et al. "Design of Asymmetrical Electron Beam Devices Using Computer Optimization," 2009 IEEE International Vacuum Electronics Conference, Rome, Italy, April 2009.

[3] L. Ives, et al, "Design of a a Doubly Convergent Multiple Beam Gun," Eleventh IEEE Intern. Vacuum Elect. Conf., Monterey, CA May 2010.

[4] T. Bui, et al. "An Optimizer for Beam Optics Analyzer, 2009 IEEE International Vacuum Electronics Conference, Rome, Italy, April 2009.

[5] Electron Gun for a Multiple Beam Klystron with Magnetic Compression of the Electron Beams, PTO filing date Feb 12, 2010, PTO S/N: 12/705,160.

[6] Thuc Bui, R. Lawrence Ives, "Nonlinear 3D magnetostatic Solver in Beam Optics Analysis," 2008 IEEE International Vacuum Electronics Conference, Monterey, CA, April 2008, pp. 461-462.

[7] J. David, R.L. Ives, H.T. Tran, T. Bui, and M. Read, "Computer Optimized Gun Design,” IEEE Trans. Plasma Sci., Vol. 36, No. 1, February 2008, pp. 156-168.

[8] L. Ives, M. Miram, A. Krasnykh, Valentin Ivanov, "Confined Flow Multiple Beam Guns for High Power RF Applications,” 13th IEEE Conf. Plasma Sci., Las Vegas, NM, June 2001.

[9] R. Lawrence Ives, G. Miram, A. Krasnykh, "Electron Gun for Multiple Beam Klystron Using Magnetic Focusing," U.S. Patent Number 6,768,265, July 27, 2004.

[10] R. Lawrence Ives, George Miram, "Electron gun for a multiple beam klystron using magnetic focusing with a magnetic field corrector," U.S. Patent No. 6,847,168, Jan. 25, 2005.

[11] R. Lawrence Ives, Adam Attarian, William Tallis, Cynthia Andujar, Virginia Forstall, Hien Tran, Michael Read, Thuc Bui, "Design of Doubly Convergent 
Multiple Beam Gun,” IEEE Trans. Plasma Sci., Special Edition on High Power Microwave Sources, Vol. 38, No. 6, pp. 1337-1344, June 2010.

[12] R. L. Ives, et al., "Computer Optimized Design of 3D Electron Beam Devices," 34th Intern. Conf. on Infrared, Millimeter, and Terahertz Waves, Busan, Korea, September 2009.

[13] T. Bui, et al, "Recent Advances in Beam Optics Analyzer," 35th Intern. Conf. on Infrared, Millimeter, and Terahertz Waves, Rome, Italy, September 2010.

[14] L. Ives et al, "Optimization for the Design of Electron Guns," 37th Intern. Conf. on Plasma Science, Norfolk, VA, June 2010.

[15] R.L. Ives, Adam Attarian, Thuc Bui, Michael Read, John David, Hien Tran, William Tallis, Steven Davis, Sean Gadson, Noah Blach, David Brown, Erin Kile, "Computational Design of Asymmetrical Electron Beam Devices," IEEE Trans.

Plasma Sci., Vol. 56, No. 5, May 2009, pp. 753-761. 